\title{
Excellent Response to Anti-PD-1 Therapy in a Patient with Hepatocellular Carcinoma Intolerant to Sorafenib
}

\author{
Christoph Roderburg $^{\mathrm{a}} \quad$ Marie-Luise Berres $^{\mathrm{a}} \quad$ Alexander Wree $^{\mathrm{a}}$ \\ Sven H. Loosen ${ }^{a}$ Tom Luedde ${ }^{a}$ b Christian Trautwein ${ }^{a}$ \\ a Department of Medicine III, University Hospital RWTH Aachen, Aachen, Germany; \\ ${ }^{b}$ Division of Gastroenterology, Hepatology and Hepatobiliary Oncology, Aachen, Germany
}

Keywords

Hepatocellular carcinoma · Nivolumab · PD-L1 · Response

\section{Abstract}

Background: Hepatocellular carcinoma (HCC) represents the most common primary carcinoma of the liver. Most patients present with advanced or metastatic HCC at diagnosis and face a very limited prognosis. Systemic treatment with the tyrosine-kinase inhibitors sorafenib or lenvatinib is considered as the treatment of choice in these patients. In patients intolerant to tyrosine-kinase inhibitors, no standard therapy is approved so far, but several agents have demonstrated efficacy in clinical trials. As such, the checkpoint inhibitors nivolumab and pembrolizumab have been shown to induce tumor response and to prolong survival in patients with advanced $\mathrm{HCC}$, both when intolerant to sorafenib or after failure of a first-line therapy with sorafenib. Case Report: We here report the case of a patient with advanced HCC that demonstrated severe toxicity upon first-line treatment with sorafenib. Administration of sorafenib was discontinued and after careful consideration the patient started a second-line treatment with the PD-1 antibody nivolumab. Strikingly, this treatment led to a significant regression of tumor size and a drastic reduction of alpha-fetoprotein serum concentrations. At 17 months after initiation of treatment, the patient is still alive in excellent condition with sustained tumor response. Conclusion: In summary, we report on a very rare case of a patient with HCC demonstrating an almost complete response to checkpoint inhibitor treatment.

(c) 2019 S. Karger AG, Basel

\section{Introduction}

Hepatocellular carcinoma (HCC) represents the most common primary malignancy of the liver. In almost all cases HCC occurs in the setting of chronic liver injury and liver cirrhosis. In the last decade, HCC has risen to become the fifth most common cause of cancer and the second leading cause of cancer-related death worldwide [1]. The incidence of HCC varies from 3/100,000 in Western countries to 78/100,000 in Africa and Asia, mapping the geographical distribution of its most important risk factors, i.e., viral hepatitis $\mathrm{B}(\mathrm{HBV})$ and hepatitis $\mathrm{C}(\mathrm{HCV})[2,3]$. Besides viral hepatitis and alcoholic liver disease, metabolic diseases such as diabetes mellitus type 2 and nonalcoholic fatty liver disease/nonalcoholic steatohepatitis have emerged as risk factors that are increasingly prevalent, especially in the Western world [4].

Therapeutic management of HCC is dependent on the extent of the tumor and the degree of liver dysfunction. While in patients with early tumor stages, surgical resection, orthotopic liver transplantation, or ablative therapies might offer the chance for cure, approximately $50 \%$ of patients are diagnosed with locally advanced or metastatic disease and, therefore, are not eligible for these potentially curative treatments $[2,3]$ but should receive systemic therapy. Based on the results of the SHARP study, sorafenib was established for almost a decade as the sole systemic treatment for these patients [5]. However, in clinical routine, sorafenib is associated with

\section{KARGER}

(c) 2019 S. Karger AG, Basel
Univ. Prof. Dr. med. Christian Trautwein

Department of Medicine III, University Hospital RWTH Aachen

Pauwelsstrasse 30

DE-52074 Aachen (Germany)

E-Mail ctrautwein@ukaachen.de 

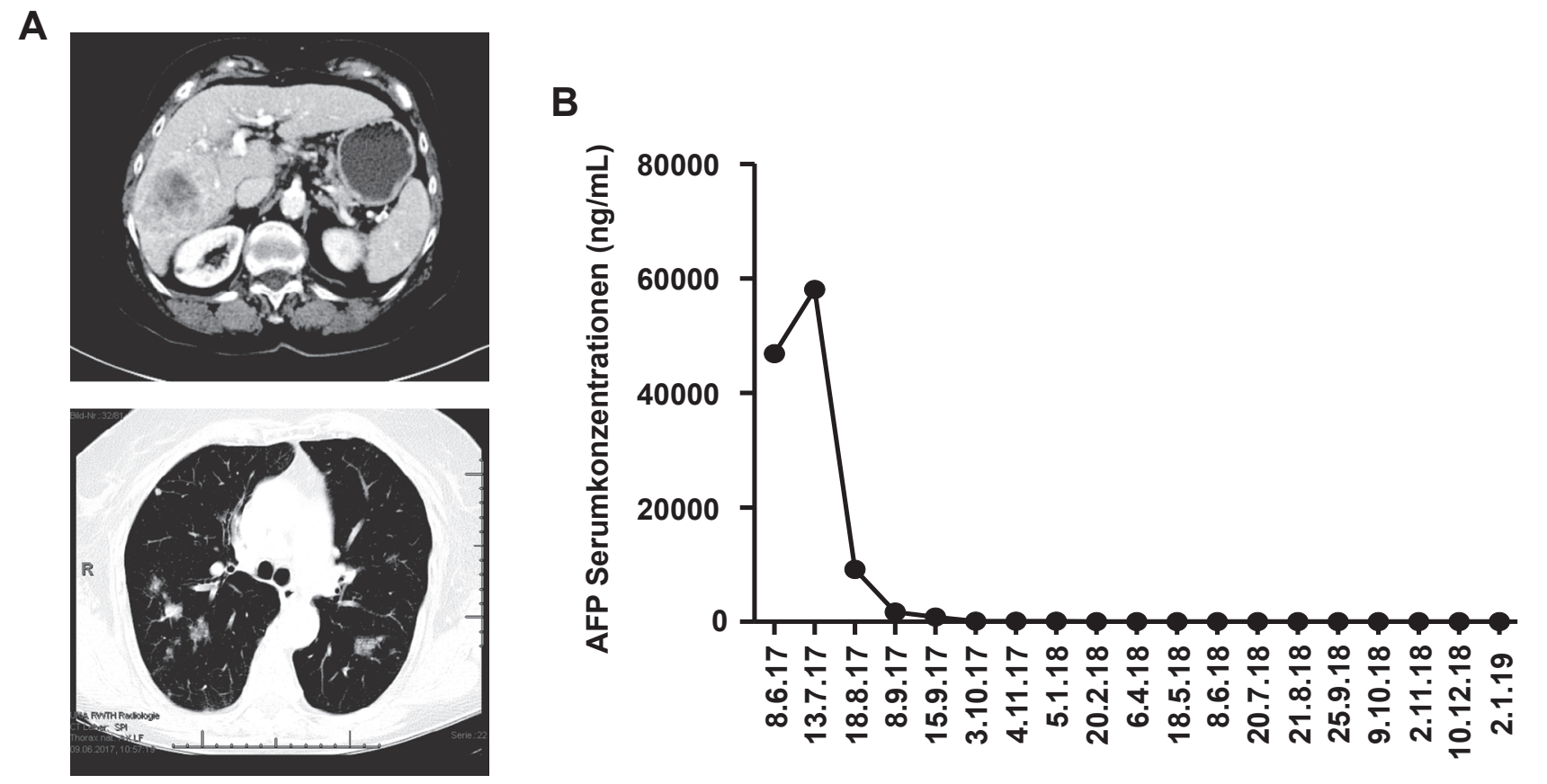

Fig. 1. A CT scan showing representative liver lesions at baseline (upper image); CT scan showing lung metastases at baseline (lower image). B Graphical depiction of changes in the alpha-fetoprotein serum concentrations over time.

significant toxicities such as hand-foot syndrome, fatigue, and gastrointestinal side effects. Only recently, lenvatinib was introduced as an alternative first-line treatment option in the context of advanced or metastasized HCC. After failure of a first-line therapy, regorafenib and, in the case of patients with a baseline alphafetoprotein (AFP) $>400 \mathrm{ng} / \mathrm{mL}$, ramucirumab have demonstrated efficacy in the RESOURCE and REACH-2 trials, respectively [6].

In recent years, immunotherapy in the form of immune checkpoint blockade has initiated a paradigm shift in cancer treatment [7]. Blockade of immune checkpoint pathways such as the programmed cell death receptor-1 (PD-1) pathway or the cytotoxic T-lymphocyte antigen-4 (CTLA-4) pathway can potentially offer a treatment strategy to reinstate host immune response against HCC and ultimately tumor regression [8]. Just recently, both the CheckMate 040 trial and the KEYNOTE-224 trial, large single-arm phase I/II trials, have reported promising results for nivolumab and pembrolizumab when used as salvage therapy after failure of a sorafenibbased first-line therapy in patients with advanced or metastatic HCC $[9,10]$. In both trials, fatigue, pruritus, and rash embodied the most prevalent adverse events. Notably, the expression of programmed cell death ligand 1 (PD-L1) on the tumor cell surface was not found to be predictive for treatment response or patients' survival.
We report a case of a patient with HCC that demonstrated an excellent response to treatment with nivolumab after being intolerant to a first-line therapy with sorafenib.

\section{Case Report}

We report the case of a 77-year-old female patient with chronic HCV-associated liver cirrhosis. Chronic HCV infection (genotype 1b) was first diagnosed as non-A, non-B hepatitis in 1995, and the patient was later treated with pegylated interferon- $\alpha 2 \mathrm{a}$ in combination with ribavirin, but did achieve a sustained virological response. A DAA treatment had yet not been initiated for unknown reason.

In June 2017, the patient presented at our outpatient unit with worsening of her general condition and was found to have a large heterogeneous irregular mass within the right hepatic lobe on ultrasound scan. A subsequent multi-slice CT scan revealed a diffuse bilobular liver carcinoma accompanied by disseminated lung metastases (Fig. 1A). Moreover, partial portal vein thrombosis was diagnosed. Serum AFP was $58.287 \mathrm{ng} / \mathrm{mL}$ at that time (Fig. 1B). Because of the radiologic characteristics of the liver lesions for HCC (hyper-enhancement during arterial phase and early washout in venous phase) we refrained from performing a tumor biopsy. Liver function was well preserved (Child-Pugh score 5), thus, a tumor stage of BCLC C according to the Barcelona Clinic Liver Cancer (BCLC) classification was assigned.

Based on the results of the SHARP study, we initiated a treatment with sorafenib. Considering her deteriorated general condition, we started the treatment with a reduced dose (200 mg b.i.d.). 
Nevertheless, the patient rapidly experienced extremely painful skin alterations (peeling, blisters, and hyperkeratosis) limiting selfcare activities of daily life, leading to the diagnosis of a severe handfoot skin reaction (CTC grade 4). Since clinical symptoms further worsened despite maximal supportive care and temporary discontinuation of sorafenib, the treatment was finally withdrawn after 1 cycle of therapy in June 2017. At that time, published data from the CheckMate 040 trial, a phase $1 \mathrm{~b} / 2$ trial analyzing the efficacy of the immune checkpoint PD-1 inhibitor nivolumab in patients with advanced HCC were available [9]. In this trial, including a total of 262 patients ( 48 patients in a dose escalation phase and 214 in a dose expansion phase), encouraging response rates of $20 \%$ were reported, independent of the presence of HCV infection. Based on these results, after careful consideration and informed patient consent, we initiated a treatment with nivolumab $(3 \mathrm{mg} / \mathrm{kg}$ body weight biweekly) in July 2017. The treatment was initially well tolerated and already after 4 cycles of therapy we observed a striking decrease in serum AFP concentrations (Fig. 1B). At the same time, a consecutive CT scan confirmed a significant shrinkage of tumor mass. Hence, nivolumab treatment was continued. Besides a lowgrade RASH (CTCAE grade 1) localized at the lumber area, no significant toxicity occurred during the course of further treatment. Imaging after 8 cycles as well as after 12 cycles of nivolumab ( 4 and 6 months, respectively) revealed a further decrease in size of the liver lesions, and AFP declined to almost normal levels (Fig. 1B). Unexpectedly, after the 12th cycle of therapy (March 2018), the patient developed shiny, red, flat-topped papules as well as vesicles and bullae on both legs and feet. Based on histopathological analysis from skin biopsies the diagnosis of a lichen ruber planus aggravated by a bacterial superinfection was made, leading to an unplanned hospitalization and the need for both intravenous antibiosis and the administration of prednisolone. Unfortunately, the healing process was significantly prolonged so that nivolumab treatment could not be resumed until July 2018. At this time point a slight tumor progression was apparent in the CT scan, while AFP levels remained almost unchanged (Fig. 1B). After extensive discussion of the chances and risks of a re-induction of nivolumab, we re-initiated therapy in August 2018. Since then a further $9 \mathrm{cy}-$ cles of nivolumab were given and well tolerated. Tumor size and AFP levels remained constant during this whole period, confirming the presence of a sustained response under therapy with nivolumab in this patient.

\section{Discussion/Conclusion}

We report a case of a patient with HCC with excellent response to nivolumab after being intolerant to a firstline therapy with sorafenib. Although HCC is the second most frequent cause of cancer mortality worldwide, only few options for the treatment of advanced HCC are available. Sorafenib has been tested in the SHARP trial, where it has shown improvement in median OS from 7.9 to 10.7 months [5]. However, treatment is often complicated by severe treatment-related toxicities. For patients who do not tolerate tyrosine kinase inhibitor therapy, no therapeutic options were available for a long period of time. Only recently the CheckMate 040 trial provided evidence for the use of nivolumab, a fully humanized IgG4 monoclonal antibody to $\mathrm{PD}-1$, in patients intolerant to sorafenib [9].
In recent years, immune checkpoint blockade has changed our view on the treatment of a large number of different malignancies. PD-1, described in 1992 by Honjo and colleagues [11], represents the most prominent checkpoint receptor so far. Blocking the interaction between PD-1 and its ligand PD-L1 resulted in an enhanced and robust immune response leading to a marked increase in anticancer activity in different experimental cancer models. Based on these findings, the humanized anti-PD-1 antibody nivolumab was tested in a broad variety of clinical trials for its efficacy in the therapy of malignant tumors. Nivolumab is currently approved for the treatment of, for example, melanomas, non-small-cell lung cancer, and kidney cancer. Besides nivolumab, other PD-1/PD-L1 antibodies have shown comparable effects in manifold cancer entities. In the context of HCC, long-awaited results on the efficacy of checkpoint inhibition have recently been published $[9,10]$. In the CheckMate 040, an open-label, noncomparative, phase $1 / 2$ dose escalation and expansion trial including patients with histologically confirmed advanced HCC, nivolumab demonstrated objective response rates of about $20 \%$, both in patients that were pre-treated with sorafenib and in therapy-naïve patients. Here we describe the case of a patient that was treated with nivolum$\mathrm{ab}$ after being intolerant to a first-line therapy with sorafenib. The patient demonstrated an early and excellent response lasting now for $>18$ months. Notably, this clinical course is in line with data from the CheckMate 040 trial [9], where responses occurred early in treatment and were long-lasting (median duration of response 17 months). Only recently, different groups identified the presence of $\mathrm{HCV}$ as a positive predictor for sorafenib efficacy in patients with HCC [12]. In line with this, we initiated a first-line therapy with sorafenib in our patient suffering from HCV-related liver cirrhosis. In the CheckMate 040 trial, a total of 214 advanced HCC patients were treated in the dose expansion phase: 56 patients were not infected with $\mathrm{HCV}$ or HBV and were sorafenib treatmentnaïve or intolerant to sorafenib; 57 patients exerted disease progression following sorafenib; 50 patients were infected with HCV; and 51 were infected with HBV [9]. Despite the fact that this study was not powered for statistical comparisons between the different subgroups and similar response rates were observed in all patient groups, survival was numerically higher in patients who had not previously been treated with sorafenib or were intolerant (compared to progressors), supporting the treatment sequence reported here.

The safety profile of nivolumab is widely considered as manageable. In the CheckMate 040 trial, grade 3/4 treatment-related adverse events were seen in 40 (19\%) patients and grade $3 / 4$ treatment-related serious adverse events were seen in $9(4 \%)$ patients. Skin-related toxicities represented the most frequent adverse events. Interest- 
ingly, our patient developed a lichen ruber planus 4 months after treatment initiation with nivolumab. In line with our report, Lindner et al. [13], in 2009, reported the case of a metastatic renal cell carcinoma patient who developed an "immune-mediated" lichen ruber 6 months after starting nivolumab therapy. Later, various groups reported the occurrence of lichenoid lesion during antiPD-1/anti-PD-L1 therapy [14, 15]. Additionally, in our patient the development of lichen ruber might be related to the infection with $\mathrm{HCV}$, representing an important risk factor for this disease [16]. After dissolving of symptoms, re-initiation of therapy in our patient did not induce recurrence of lichen, but again induced stabilization of tumor growth after progression during treatment discontinuation. Therefore, our case might implicate that reinitiation after dissolving side effects might be safe and efficient again, as recently suggested in melanoma [17]. Moreover, the development of a severe immune-mediated cutaneous toxicity upon nivolumab treatment in a patient demonstrating an excellent response to therapy further underscores recent data suggesting that immune-related adverse events predict the therapeutic efficacy of anti-PD-1 antibodies in cancer patients [18].
In summary, we present a case of HCV-related HCC successfully treated with the anti-PD-1 antibody nivolum$\mathrm{ab}$ after being intolerant to a first-line therapy with sorafenib. Together with many other reports and clinical trials, this case supports the efficacy of sequential therapies in the treatment of patients with advanced HCC.

\section{Statement of Ethics}

The patient described in this paper gave written informed consent to publish her case (including publication of images).

\section{Disclosure Statement}

The authors have no conflicts of interest to declare. No funding was received for this study.

\section{Author Contributions}

All authors were involved in the treatment of the described patient. All authors read and approved the final paper.

\section{References}

1 Wallace MC, Preen D, Jeffrey GP, Adams LA. The evolving epidemiology of hepatocellular carcinoma: a global perspective. Expert Rev Gastroenterol Hepatol. 2015 Jun;9(6):76579.

2 Forner A, Reig M, Bruix J. Hepatocellular carcinoma. Lancet. 2018 Mar;391(10127):130114.

3 European Association for the Study of the Liver. EASL Clinical Practice Guidelines: management of hepatocellular carcinoma. J Hepatol. 2018 Jul;69(1):182-236

4 Degasperi E, Colombo M. Distinctive features of hepatocellular carcinoma in non-alcoholic fatty liver disease. Lancet Gastroenterol Hepatol. 2016 Oct;1(2):156-64

5 Llovet JM, Ricci S, Mazzaferro V, Hilgard P, Gane E, Blanc JF, et al.; SHARP Investigators Study Group. Sorafenib in advanced hepatocellular carcinoma. N Engl J Med. 2008 Jul; 359(4):378-90.

6 Llovet JM, Montal R, Sia D, Finn RS. Molecular therapies and precision medicine for hepatocellular carcinoma. Nat Rev Clin Oncol. 2018 Oct;15(10):599-616

7 Alsaab HO, Sau S, Alzhrani R, Tatiparti K, Bhise K, Kashaw SK, et al. PD-1 and PD-L1 checkpoint signaling inhibition for cancer immunotherapy: mechanism, combinations, and clinical outcome. Front Pharmacol. 2017 Aug;8:561.
8 Kudo M. Immune Checkpoint Inhibition in Hepatocellular Carcinoma: Basics and Ongoing Clinical Trials. Oncology. 2017;92 Suppl 1:50-62.

9 El-Khoueiry AB, Sangro B, Yau T, Crocenzi TS, Kudo M, Hsu C, et al. Nivolumab in patients with advanced hepatocellular carcinoma (CheckMate 040): an open-label, noncomparative, phase $1 / 2$ dose escalation and expansion trial. Lancet. 2017 Jun;389(10088): 2492-502.

10 Zhu AX, Finn RS, Edeline J, Cattan S, Ogasawara S, Palmer D, et al.; KEYNOTE-224 investigators. Pembrolizumab in patients with advanced hepatocellular carcinoma previously treated with sorafenib (KEYNOTE-224): a non-randomised, open-label phase 2 trial. Lancet Oncol. 2018 Jul;19(7):940-52.

11 Ishida Y, Agata Y, Shibahara K, Honjo T. Induced expression of PD-1, a novel member of the immunoglobulin gene superfamily, upon programmed cell death. EMBO J. 1992 Nov; 11(11):3887-95

12 Bruix J, Cheng AL, Meinhardt G, Nakajima K, De Sanctis Y, Llovet J. Prognostic factors and predictors of sorafenib benefit in patients with hepatocellular carcinoma: analysis of two phase III studies. J Hepatol. 2017 Nov; 67(5):999-1008

13 Lindner AK, Schachtner G, Tulchiner G, Staudacher N, Steinkohl F, Nguyen VA, et al. Immune-related lichenoid mucocutaneous erosions during anti-PD-1 immunotherapy in metastatic renal cell carcinoma - A case report. Urol Case Rep. 2018 Nov;23:1-2.
14 Schaberg KB, Novoa RA, Wakelee HA, Kim J, Cheung $C$, Srinivas $S$, et al. Immunohistochemical analysis of lichenoid reactions in patients treated with anti-PD-L1 and anti-PD-1 therapy. J Cutan Pathol. 2016 Apr;43(4):33946.

15 Shi VJ, Rodic N, Gettinger S, Leventhal JS, Neckman JP, Girardi M, et al. Clinical and Histologic Features of Lichenoid Mucocutaneous Eruptions Due to Anti-Programmed Cell Death 1 and Anti-Programmed Cell Death Ligand 1 Immunotherapy. JAMA Dermatol. 2016 Oct;152(10):1128-36.

16 Pinelli S, Basile S, Benedetti Panici P, D’Erme AM, Romanelli M, Plotti F, et al. Association between HCV infection and cutaneous-mucosal lichen planus: an update. Eur J Dermatol. 2017 Jun;27(3):329-31.

17 Koppolu V, Rekha Vasigala VK. Checkpoint immunotherapy by nivolumab for treatment of metastatic melanoma. J Cancer Res Ther. 2018 Oct-Dec;14(6):1167-75.

18 Rogado J, Sánchez-Torres JM, Romero-Laorden N, Ballesteros AI, Pacheco-Barcia V, Ramos-Leví A, et al. Immune-related adverse events predict the therapeutic efficacy of antiPD-1 antibodies in cancer patients. Eur J Cancer. 2019 Jan;109:21-7. 